\title{
Selected issues of the Bluetooth techno- logy in control on-line and telemetry
}

\author{
Elzbieta Grzejszczyk Assosciate Professor, Ph. D. eng. \\ Warsaw University of Technology, Faculty of Electrical Engineering, Warsaw, Poland
}

\begin{abstract}
This paper discusses how Bluetooth networks are built and facilitate communication. Analysis of protocol stack and selected profiles application were presented. A remote measurements and on-line controlling system for a given object was described as practical implementation of the technology standard. The developed software being an example of utilising the Bluetooth technology in telemetry is a substantial part of this paper.

The article concludes with didactic aspects of discussed solutions.
\end{abstract}

Keywords: Remote controll, communication on-line, Bluetooth standard, remote measurements.

\section{Bluetooth technology}

\subsection{Introduction}

Over recent years, the wireless technology area has been booming $[1,2,3]$. Bluetooth wireless technology standard was developed as well. It uses radio waves in the frequency band 2.45 $\mathrm{GHz}$ for exchanging information between devices (the IEEE 802.15.1 standard). Communication over Bluetooth is easy to establish and the networks themselves are easily set-up as well. They do not require any input from the user, and are managed themselves by connected devices. (Fig.2). Among other advantages of this technology are high standardization, small transceiver microchips and low cost of mass-produced integreted chips. By combining the benefits of radio transmission and above-mentione features, Bluetooth technology has made its way into remote telemetry and control engineering, data exchange and voice transmission. However, its biggest limitation is the relatively low range. Low-power equipment can generate radio waves with $10 \mathrm{~m}$ range, whereas higherpower devices are capable of producing radio waves with range of up to $100 \mathrm{~m}$. The transmission speed reaches $3 \mathrm{Mb} / \mathrm{s}$ for the latest solutions. [5]

\subsection{Network structure and communication}

Information sent via networks Bluetooth is encoded FSK system ( Frequency-Shift Keying) - one of the oldest signal modulation techniques. FSK utilises the carrier frequency of electromagnetic wave modulated by digital information transmited through frequency changes. (rectangular signal).Devices within the Bluetooth network communicate using the star topology (Fig. 2). For data exchange employed are both the TDD (Time Division Duplex) and FHSS (Frequency - Hopping Spread Spectrum) systems.

TDD system generate 1600 time-slots per second $(1600 \times 625 \mu \mathrm{s})$.

FHSS is a method of planned frequency hopping between transmission channels $\mathrm{f}=2402 \mathrm{MHz}$ and $\mathrm{f}=2480 \mathrm{MHz}$ over 
$1 \mathrm{MHz}$ band. (Fig. 1) Devices are communicating using half-duplex method. Two fundamental protocols are used for data exchange:

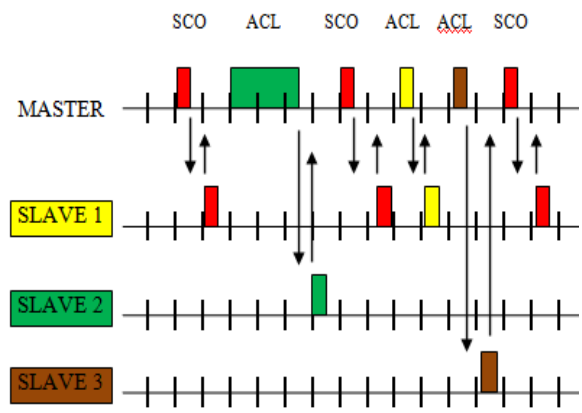

Fig.1: The example of transmission at Bluetooth networks

Mode SCO (Synchronous connectionoriented) where the Master communicates with the Slave in predefined time periods by utilising allocated timeslots.

Mode ACL (Asynchronous Connection Less) where the Master device switches between addressing consecutive devices connected to the network.

The basic network of Bluetooth standard is piconet. Piconet network set-up ad-hoc and can communicate with a maximum of 8 devices (Fig.2.)
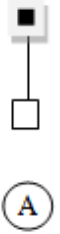
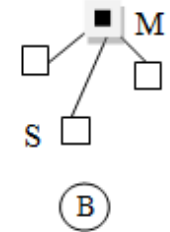

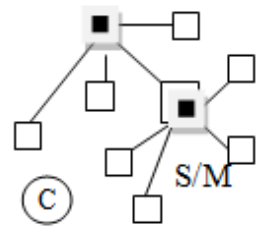

\section{M - Master S - Slave}

One device can simultaneously play the role of slave (S) in one piconet and master $(\mathrm{M})$ in the other. One device could never play the role of Master in two subpiconets. Network displayed in Fig. 2/ C illustrates a situation where two networks have 1 common node. (S/M). With assuring minimum power consumption of devices in mind (thereby the maximum batter life) four operating modes were designed. They are: Hold, Park, Sniff and Stand by.

Hold - master device connects a slave device. In this mode communication between devices is suspended and only master's clock is active. Slave device can request for the Hold mode (e.g. in order to attempt connection to another network). Data transmission is resumed, once the Master device switches the mode.

Park - in this mode the device is excluded from message exchange within the network. The device listens periodically to resynchronise with Master in order to receive broadcasts (information addressing all devices).

Sniff - device listens out for communication from the network. Time between listening is programmable and is determined by the application.

Standby - the system searches for devices not connected to the piconet. Device in the standby mode listens for network traffic each 1.28 second over 32 frequencies (consistent with hop frequencies). In case of the ACL link - device in either hold or park mode in one network, can connect to a different network.

\section{Bluetooth software and profiles}

Bluetooth software has to comply with the prerequisite of compatibility between applications and Bluetooth devices from different manufacturers. Standards define so-called application profiles using appropriate layers of the Bluetooth stack [5]

Two or more piconets can be connected together to form a Scatternet. 


\subsection{Selected profiles}

In presented application, used were PAN (Personal Area Networking Profile) and

HS (Headset Profile) profiles. The PAN profile (Personal Area Networking Profile) determines how two or more Bluetooth devices communicate with each other over an ad-hoc network (Fig.3) and how the same mechanisms could be employed to set-up connection with distant devices - via the access point (Fig.4).

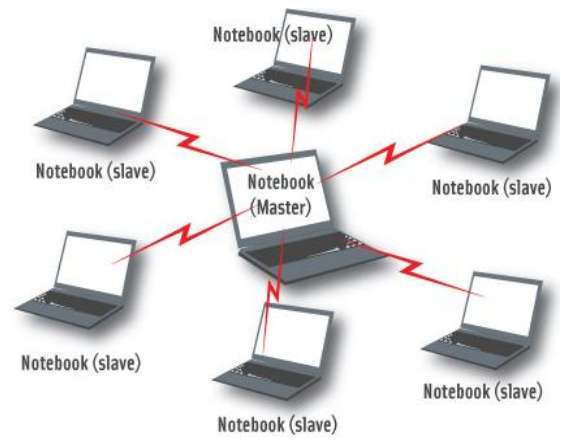

Fig.3: Single piconet personal ad-hoc network

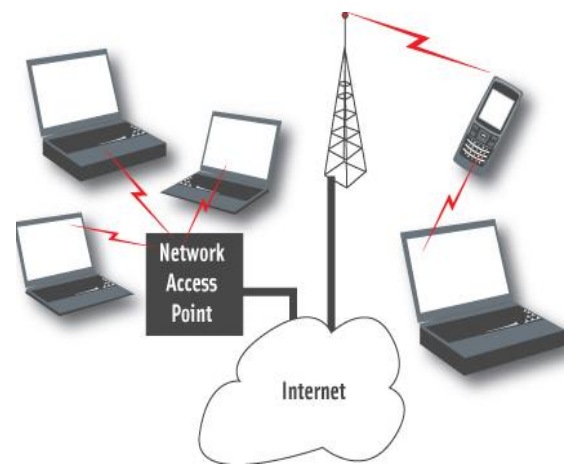

Fig. 4: Two types of network access points

Devices capable of establishing such connection are PC computers, laptops, iPads and Mobile Phones. They connect with the master either via an ad-hoc network or Access Point to other networks.

The HSP profile (Headset Profile) is a lightweight version of the PAN profile
(Personal Area Network) and an example of single piconet personal ad-hoc network. (Fig. 5). Communication between devices operating using the HS profile utilises protocol stack displayed in Fig. 6.

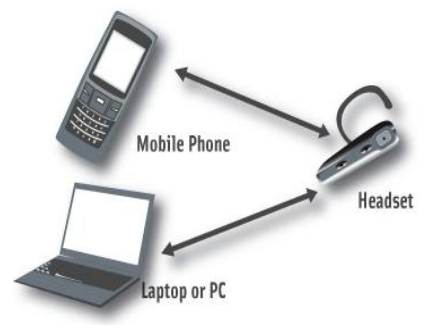

Fig 5: Typical use of the HS profile

HS data exchange model is displayed in Fig.7

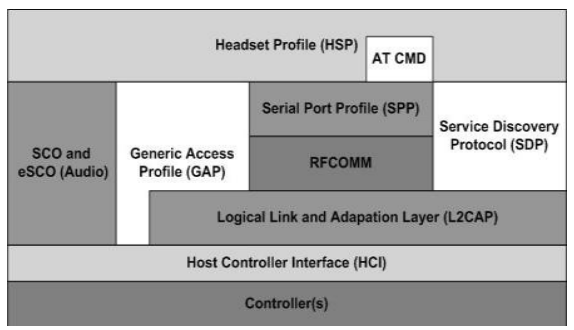

Fig. 6: Bluetooth protocol stack used in HS profiles

The HSP defines two roles, that of an Audio Gateway (AG) and a Headset(HS): (1) Audio Gateway (AG) - This is the device that is the gateway of the audio, both for input and output, typically a mobile phone or PC . (2) Headset (HS) This is the device acting as the Audio Gateway's remote audio input and output mechanism. The Baseband, LMP and L2CAP are the OSI layer 1 and 2 Bluetooth protocols. RFCOMM is the Bluetooth adaptation of GSM TS 07.10. SDP is the Bluetooth Service Discovery Protocol. Headset Control is the entity responsible for headset-specific control 


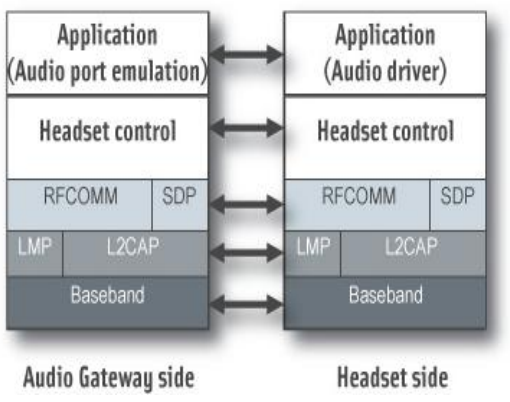

Fig. 7: Communication between devices using the HS profile

signalling [5] (The Serial Port Profile is used as base standard)

\section{Example of implementation Blue- tooth technology}

Figure 8 illustrates an example of discussed technology standard developed at the Faculty of Electrical Engineering WUT (spec. Information Technology) [4]

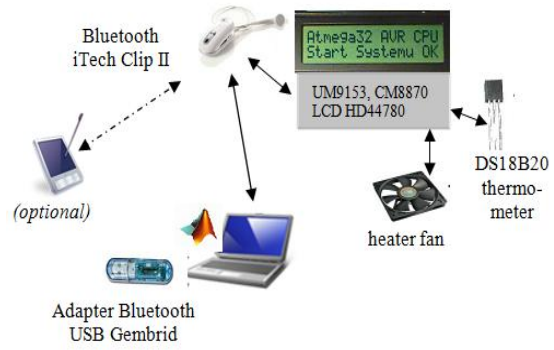

Fig.8: High-level diagram of the system and its components

The task of the system is to remotely control device in accordance with remotely taken measurement. Bluetooth is the transmission medium using a Bluetooth Adapter (data transfer speed up to 1 MBs, up to 7 devices, $20 \mathrm{~m}$ range) and the Clip II Headset Mode. Devices communicate with each other by receiving and sending DTFM signals (Time Division Multiple Access).
The system consists of two components. 1 . Master control component using a PC computer (WinXP) equipped with software developed in the Matlab 7.0 environment. $2 . \quad$ Microcontroller ( executable component) based on the ATmega 32 microcontroller from the AVR family. The microcontroller mentions information with code transmitter DTMF UM915313, code receiver DTMF CM8870, digital thermometer DS18B20, alphanumeric LCD display using the HD44780 driver and it controls MT01 computer cooler. The Microcontroller system receives control messages for the MT01 cooler, measures temperature using the thermometer DS18B20 as well sends encoded measurement results to the controlling component. (to PC computer)

The controlling component sends instructions to the microprocessor system, receives measurements and converts them into graphic representations. (Fig. 9) The operator/computer sends to the microcontroller instruction to measure temperature, then runs received reading against predefined minimum and maximum of the measuring range. Should the value exceed any end of the scale, the cooler is either activated or deactivated. System software for remote temperature monitoring and controlling comprises two closely linked - program modules.

The first module concerns the microprocessor chip software responsible for temperature monitoring and controlling the cooler. The second module concerns the user interface software (Fig.9). 


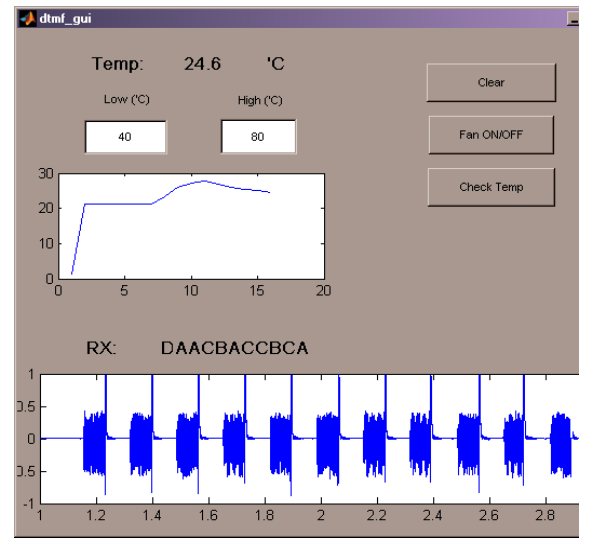

Fig. 9: Remote measurements and telemetrics system - user interface

The AVR microcontroller was programmed in $\mathrm{C}$ language. The second language was coded in Matlab 7.0.

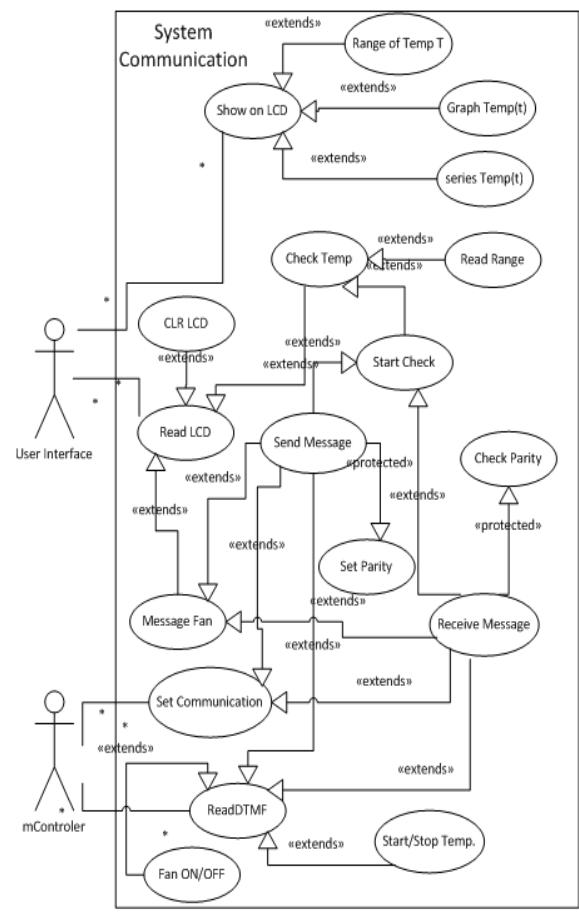

Fig: 10: Use cases for control on-line

\section{Didactic aspects of presented solu- tions}

Any cognitive process consists of two fundamental elements/levels.

Reaching of the knowledge and reaching of the skills - to deploy acquired knowledge. We expect students to reproduce the knowledge (RP ReProduce), terms and phenomena they explored and to understand them (RO understanding) (Questions: e.g. "Why do we refer to networks as self-organising networks? or - "Why we use programme profiles in the Bluetooth technology?").

As far as the skills are concerned, we expect students to be able to deploy reached knowledge in typical cases ST (Situation Typical) and problematic cases SP (Situation Problematic)

An example of typical case (ST) would be employing the Bluetooth/HS profile to set-up a typical headset - mobile phone connection. Another example is sending photos from mobile phone to computer or iPad. An example of resolving a problematic/challenging case (SP) is the above-mentioned system. Challenging of the project (SP) was to design and develop software for the system based on knowledge about the Bluetooth technology standard. Positive outcome of verifying taken assumptions/solutions, confirmed by obtained results, was conclusive as to the reaching of knowledge and skills to deploy it was successful. Teaching methodology - outlined in this paper for Bluetooth network related issues came up to expectations. Furthermore, it gives grounds for next projects. Those projects would most probably be iPads as graphical user interface replacing computer display as well as other executable systems in place of the cooler.

The final conclusion is that the key competence in using the Bluetooth technology is the ability of programming it. 


\section{References}

[1] B.Fryskowski,E. Grzejszczyk , "Sysmy transmisji danych" (Systems of Data Transmission), Wydawnictwa Komunikacji $i$ Łaczności (Transport and Communication Publishers), Warsaw, 2010, pp 174 - 203

[2] E.Grzejszczyk,"Teleservisowa komunikacja z pojazdem samochodowym" (Teleservice communication with motor vehicle), Electrical Review, ISSN 0033-2097, R. 87 NR 12a/2011, pp.94-98
[3] E.Grzejszczyk, "Analiza wybranych usług teleinformatycznych serwisu BMW" (Analysis of selected telematic services), Electrical Review, ISSN 0033-2097, R. 88 NR 7a/2012, pp. 294 - 296

[4] Thesis, Electrical Dept.,Warsaw University of Technology, Cierniak M.., "Remote control and telemetry over Bluetooth system", Warsaw 2006

[5] https://www.bluetooth.org/Technical/ Specifications/adopted.htm 